\title{
Black Spot Analysis and Solutions on State Highways at Khargone District
}

\author{
Arpit Jaiswal $^{1}$ and Atul Bhatore ${ }^{2}$
}

Abstract- India is a leading developing country and road safety is still in a premature stage. Accident severity is growing in rapid order due to increasing in vehicle population. Accident leads to disablement, damage to health and property, death of human or animals' social suffering and general degradation of environment. CAGR reports of year 2014 shows accident in rural region is $53.70 \%$ and urban region $46.30 \%$, the report clearly indicating that safety requirement are needs to be more concentrate on rural area that that of urban area. Government data shows about 400 persons where died every day in year 2015 and there is one death at every 3.75 minutes because of road accidents. The high accident rate is largely considered to the inadequacy of the highways and other main roads to meet the traffic demands, road user behaviour, vehicular defects, poor road geometrics and inadequate visibility. Road accidents results in heavy wealth loss to the country. Necessary of road safety is to reduce accident involving both human element and vehicles there by developing the road safer and user friendly to traffic.

In Madhya Pradesh state area around our district headquarter Khargone roads has major connectivity and there are not big industries but these road carries large numbers of passengers to industrial area of Indore and Gujarat through state highways and district road.

Keywords - TRAFFIC, ACCIDENT, ROAD USER, ROAD SAFETY, TRAFFIC VOLUME

\section{INTRODUCTION}

Road accident takes away the life of 3398 peoples every day. This is global humanitarian disaster, and it is man-made as per Global Road Safety Partnership Annual Report (GRSPAR-2014).Road safety is most important problem in our society. By Year -2014 rate of death in road accident is 2.24 million per year and about 50 million peoples were injured every year. About $50 \%$ of all road death is among pedestrian, cyclist or motorcycle riders. In 2014 Indian roads were at their dead list claiming more than 16 lives every hour on average. Over 1.41 lakh people died in crashes, 3\% more than that of fatalities in 2013.If the current trends of road accident continue than it is predicted to be third leading contributor to global burden of diseases and injury by 2020.now traffic accident are at 8th position globally. India having more number of fatalities recorded by road accidents in the world had earned the dubious distinction. Road safety is emerging as major social concern around the world especially in India (Shivkumar and Krishnaraj, 2012). Accidents are somewhat a drain on the national economy and may lead to disablement, damage to the health and property, death, social suffering and general environmental degradation. To minimize the no of crashes by any kind and a road safety is the severity expected to occur on the entity 
during a specific period. Accidents and the fatalities on road are also the result of reciprocity of a number of factors. Road users in India are heterogeneous in nature, ranging from pedestrians, cycles, rickshaws, animal- driven carts, hand carts ,bullock carts and tractor trolleys, to various categories of two wheelers or three wheelers, cars, buses, trucks, and multi-axle commercial vehicles etc., The vehicle population has been steadily increasing because of changes in lifestyle of society. There are development in vehicle population with limited road space which is used by a large variety of vehicles has increase the need and urgency for a well thought-out policies on the issue of road safety. In India growth of vehicle population is directly proportional to the rate of accident. Road accidents are a human tragedy, which is directly related to high human suffering. They impose a huge socio-economic cost in terms of injuries, untimely deaths, and loss of potential income. The ramifications of road accidents can be colossal and negative impact of road accident is felt not only on individuals, their health and welfare, as well as on the economy. Consequently, it has become an issue of national concern. It includes the development as well as management of road infrastructure, provision of safer vehicles, urban land use planning, legislation and law enforcement, provision of health and hospital services, child safety, mobility planning as well as its management, etc In other words, its having spans engineering aspects of both, roads as well as vehicles on one hand and the provision of health and in post-crash scenario hospital services should available for trauma cases Road accident in India.

Road safety in India is the poorest in the world. As per MORTH 2013 India has the highest numbers of accidents in the world. Awareness among road users, safe and secure design of road components is necessary to reduce accident involving both human and vehicles.

\subsection{ROAD SAFETY \& VARIOUS CAUSES OF ACCIDENT}

Road traffic safety deals with the methods and measures used for reducing the risk of a person using the road network being affected seriously may injure or killed. The users of a road include pedestrians, cyclists, motorists and passengers by on-road public transportation, mainly travel buses and trams. Best practice road safety strategies focus upon the preventive steps on serious injury and death crashes in spite of human fallibility. Safe road design is now about providing a such kind of road environment which ensures vehicle speeds will be within the human tolerances for major injury and fatal or death wherever conflict points exist.

The various causes of accidents may be due to factors listed below:-

(i) Driver

(ii) Vehicle

(iii)Environment

\section{OBJECTIVES OF WORK}

Expansion in the road network, growth in motorization and a rising population of a country contribute towards rising in numbers of road accidents, road numbers of registered motor vehicles in the country and the country's population have rising at a compound annual growth rate (CAGR) of 3.4, 9.9 and 1.6 per cent, respectively, during the decade from 2002 to 2011. During the same period, the number of road accidents in our country increased at a CAGR of $2.1 \%$. also the number of road accident fatalities and the number of persons injured in road accidents in the country between 2002 and 2012 increased by 5.8 per cent and 2.4 per cent, respectively. Very little work has been done in India to analyze accidents on two-lane roads.

The main objectives of the study work are:-

(i) To analyse the annual, monthly, variation in accident rate on selected Stretch of rural two-lane road. 
(ii) To analyse the effect of Traffic Volume, Traffic Density and Traffic capacity of roadway on accident rate on rural Two-lane road.

(iii)To analyse effect of the maintenance of road surface and cross-sectional element on rate of accident.

\section{SITE SELECTION AND SURVEY WORK}

\section{Road Selected For the Work:}
(a) Khargone to Kasrawad Road (35.6km.) (S1)
(b) Khargone to Sanawad Road (58.4km.) (S2)
(c) Khargone to Julwaniya Road (48km.) (S3)
(d) Khargone to Bhagwanpura Road (22.8km) (S4)
(e) Khargone to Bhikangaon Road $(40.1 \mathrm{~km})(\mathrm{S} 5)$

(a) Khargone to Kasrawad Road:

Khargone Kasrawad State Highway Connect Khargone to Agra-Mumbai National Highway (NH-3) at Khalghat which connect Khargone town to Indore, also kasrawad connect historical and tourist place Maheshwar and Mandleshwar, so that this road carries large amount of traffic volume like heavy vehicle, light vehicle and passenger bus, cars etc. The route named as State highway one (SH1). There are lots of spot and stretched where always accident occurs so this road selected for Project Work

\section{(b) Khargone to Sanawad Road :}

Khargone to Sanawad road is major District road which connect Khargone to Indore-Ichhapura State Highway at Sanawad, this road connect Indore and Barwah to Khargone. Now national thermal power plant (NTPC) placed along this road, three administrative blocks are directly connected to Khargone Sanawad stretch. The road carries mixed traffic flow. The district road also having problem of accident so there are certain spots are identified by police and road authority where accident occur frequently.

\section{(c) Khargone to Julwaniya Road :}

Khargone Julwaniya State Highway road (SH-26) is having connectivity with national highway Agra - Bombay (NH-3) at Julwaniya. This road connects Khargone and other places to historical and tourist places Oon, there are Pawagiri temple for Jain and other lots of small temple and famous Mahalaxmi mandir. There are flow pattern is mixed type largely heavy vehicles are carries goods and other supplies through this stretch to Khargone Town. There lots of spots on tongue of peoples where accidents frequently occurs like barud fata, Oon, Jamothi and stretch selected for the project Work.

\section{(d) Khargone to Bhagwanpura Road :}

Khargone to Road connects Khargone to the Maharashtra state through Bistan junction, this is state highway SH-1 and the stretch up to the Bistan is critical where lots of accidents occur. Traffic volume is very high as compare to normal traffic because there is lots of traffic is connected to Maharashtra through Pal Border, there are also lots of accidents occurs.

\section{(e) Khargone to Bhikangaon Road:}

Khargone to Bhikangaon Road is major road of Khargone District which connects Khargone to Khandwa and also Indore - Ichhapur at Deshgaon. The road connected through three blocks of 
Khargone, carries a large amount of traffic. The road is state highway SH-26.here also a lots of spot where accidents are frequent.

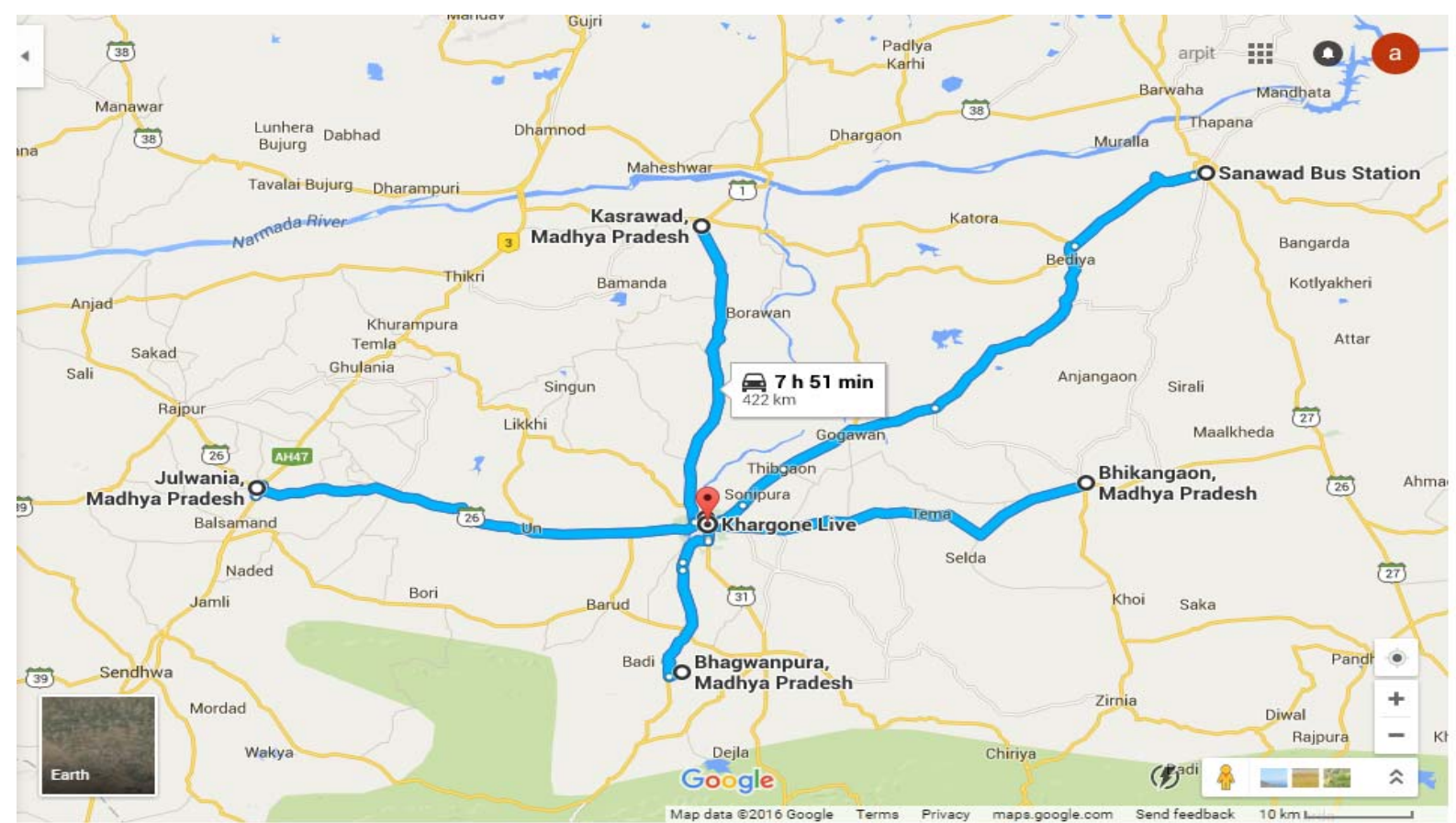

Fig.3.1:- Work Stretch, Source: - Google Map

\section{DATA COLLECTION}

Table 4.1 Details of Accident of Khargone District (2003-14)

\begin{tabular}{|l|l|l|l|l|l|}
\hline S.NO. & YEAR & FATAL & MAJOR & MINOR & TOTAL \\
\hline 1 & 2003 & 67 & 175 & 307 & 549 \\
\hline 2 & 2004 & 83 & 196 & 367 & 646 \\
\hline 3 & 2005 & 98 & 139 & 395 & 632 \\
\hline 4 & 2006 & 127 & 169 & 389 & 685 \\
\hline 5 & 2007 & 118 & 210 & 489 & 817 \\
\hline 6 & 2008 & 115 & 230 & 564 & 909 \\
\hline 7 & 2009 & 169 & 218 & 565 & 952 \\
\hline 8 & 2010 & 173 & 252 & 553 & 978 \\
\hline 9 & 2011 & 224 & 237 & 525 & 986 \\
\hline 10 & 2012 & 200 & 241 & 512 & 953 \\
\hline 11 & 2013 & 229 & 242 & 616 & 1087 \\
\hline 12 & 2014 & 215 & 273 & 702 & 1190 \\
\hline TOTAL & & 1818 & 2582 & 5984 & 10384 \\
\hline
\end{tabular}


Table 4.2 Details of Accident of Stretches Selected For the Work (2003-2015)

\begin{tabular}{|c|c|c|c|c|c|c|c|c|c|c|c|c|c|c|c|c|c|}
\hline \multirow{2}{*}{$\begin{array}{l}\text { S.NO } \\
\cdot\end{array}$} & \multirow{2}{*}{$\begin{array}{l}\text { YEA } \\
\text { R }\end{array}$} & \multicolumn{5}{|c|}{ FATAL } & \multicolumn{5}{|c|}{ MAJOR } & \multicolumn{5}{|c|}{ MINOR } & \multirow{2}{*}{$\begin{array}{l}\text { TOTA } \\
\text { L }\end{array}$} \\
\hline & & S1 & $\mathrm{S} 2$ & $\begin{array}{l}S \\
3 \\
\end{array}$ & \begin{tabular}{|l|}
$S$ \\
4 \\
\end{tabular} & $\begin{array}{l}\mathrm{S} \\
5 \\
\end{array}$ & S1 & S2 & $\begin{array}{l}\mathrm{S} \\
3 \\
\end{array}$ & $\begin{array}{l}\mathrm{S} \\
4 \\
\end{array}$ & S5 & S1 & S2 & S3 & S4 & S5 & \\
\hline 1 & 2003 & 0 & 9 & 3 & 2 & 3 & 7 & 13 & 6 & 3 & 4 & 17 & 46 & 13 & 10 & 15 & 151 \\
\hline 2 & 2004 & 4 & 6 & 3 & 1 & 2 & 6 & 14 & 7 & 1 & 11 & 32 & 51 & 12 & 21 & 18 & 189 \\
\hline 3 & 2005 & 2 & 6 & 3 & 3 & 4 & 7 & 6 & 4 & 2 & 8 & 17 & 18 & 6 & 15 & 17 & 118 \\
\hline 4 & 2006 & 5 & 5 & 3 & 2 & 7 & 15 & 19 & 4 & 6 & 5 & 30 & 29 & 7 & 25 & 20 & 182 \\
\hline 5 & 2007 & 3 & 10 & 4 & 2 & 4 & 23 & 26 & 5 & 2 & 11 & 24 & 34 & 14 & 17 & 17 & 182 \\
\hline 6 & 2008 & 3 & 7 & 5 & 4 & 5 & 17 & 38 & 1 & 0 & 11 & 26 & 39 & 21 & 28 & 19 & 224 \\
\hline 7 & 2009 & 2 & 15 & $\begin{array}{l}1 \\
6 \\
\end{array}$ & 7 & $\begin{array}{l}1 \\
0\end{array}$ & 9 & 32 & 7 & 1 & 3 & 18 & 44 & 23 & 16 & 27 & 230 \\
\hline 8 & 2010 & 6 & 9 & 7 & 1 & 8 & 18 & 28 & 5 & 4 & 3 & 40 & 36 & 24 & 21 & 21 & 231 \\
\hline 9 & 2011 & 13 & 11 & $\begin{array}{l}1 \\
4 \\
\end{array}$ & 4 & 7 & 20 & 17 & 9 & 2 & 8 & 35 & 49 & 30 & 16 & 18 & 253 \\
\hline 10 & 2012 & 19 & 9 & 7 & 7 & 9 & 12 & 19 & $\begin{array}{l}1 \\
0\end{array}$ & 7 & 9 & 27 & 38 & 19 & 9 & 14 & 215 \\
\hline 11 & 2013 & 7 & 18 & $\begin{array}{l}1 \\
1\end{array}$ & 6 & $\begin{array}{l}1 \\
3\end{array}$ & 17 & 28 & 6 & 9 & 13 & 32 & 37 & 21 & 19 & 14 & 251 \\
\hline 12 & 2014 & 17 & 12 & $\begin{array}{l}1 \\
1 \\
\end{array}$ & 7 & 9 & 20 & 22 & 6 & 2 & 14 & 30 & 45 & 20 & 27 & 15 & 267 \\
\hline 13 & 2015 & 13 & 15 & 9 & 5 & 8 & 11 & 21 & $\begin{array}{l}1 \\
4\end{array}$ & & 6 & 16 & 43 & 27 & 17 & 16 & 238 \\
\hline \multicolumn{2}{|c|}{ TOTAL } & 94 & $\begin{array}{l}13 \\
2\end{array}$ & $\begin{array}{l}9 \\
6\end{array}$ & $\begin{array}{l}5 \\
1\end{array}$ & $\begin{array}{l}8 \\
9\end{array}$ & $\begin{array}{l}18 \\
2\end{array}$ & $\begin{array}{l}28 \\
3\end{array}$ & $\begin{array}{l}8 \\
4\end{array}$ & 0 & $\begin{array}{l}10 \\
6\end{array}$ & $\begin{array}{l}34 \\
4\end{array}$ & $\begin{array}{l}50 \\
9\end{array}$ & $\begin{array}{l}23 \\
7\end{array}$ & $\begin{array}{l}24 \\
1\end{array}$ & $\begin{array}{l}23 \\
1\end{array}$ & 2745 \\
\hline
\end{tabular}

\section{ANALYSIS OF DATA}

\subsection{Annual Variation in Accidents}

\section{(a) Total Stretch Variation}

Figure 5.1(a) shows the annual variation in accident of total stretches during year 2003-2014. It is observed that the percentage accidents are increasing relatively in most of the year. In year 2014 accident rate was high and low in the year 2003. There only during 2012-13 small falls in rate of accident. It is due to increase in vehicles population, bad traffic environment, and improper road conditions and increase in population. The accident is increasing year by year, largest accident recorded in year 2014 and lowest in 2004. There are road development work in district was started after 2005 before that road condition very poor and average speed very low.

Figure 5.1(b) indicating the variation in accident from year 2003 to 2015 and there are large fall in year 2005 noted, in that duration road pavement condition is very poor and most of stretches are under construction, otherwise rate of accident are of Increasing order. 


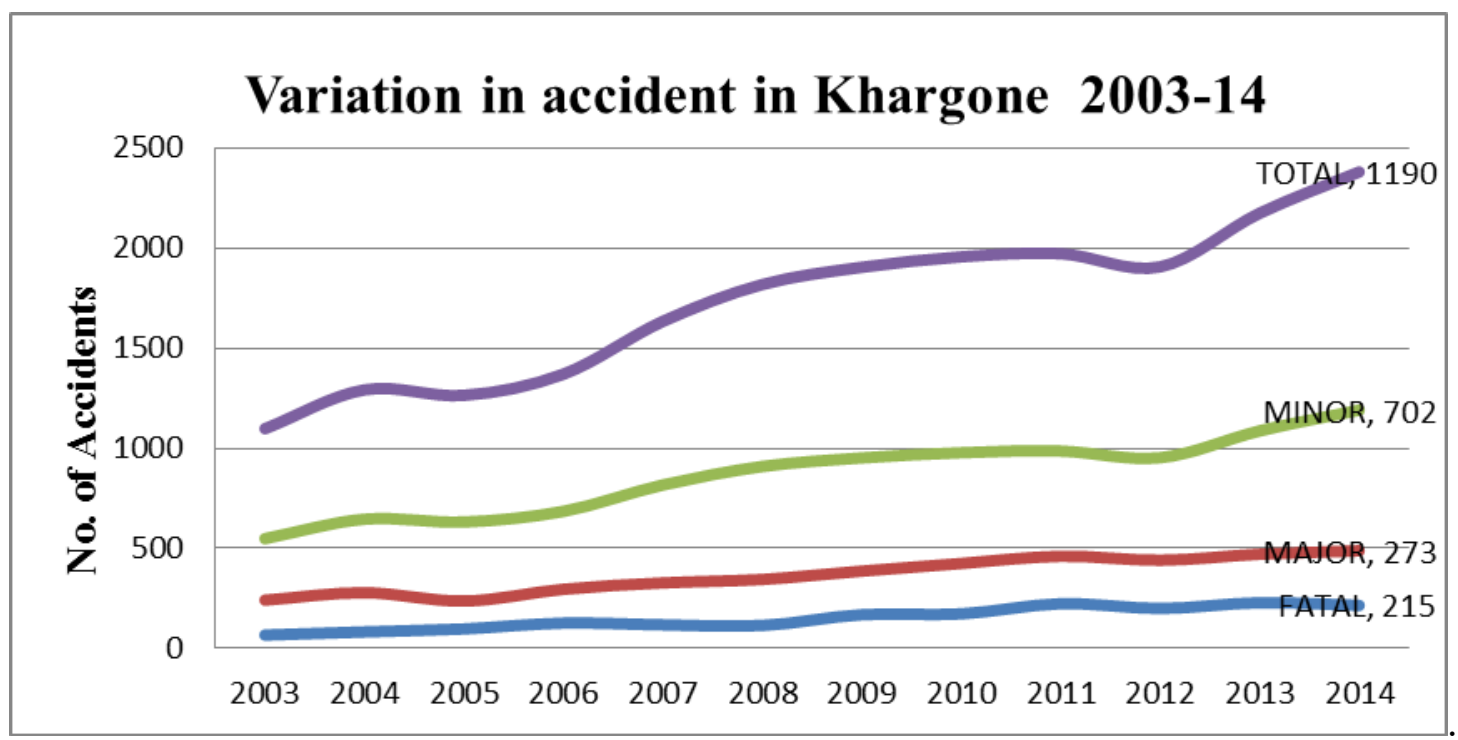

Figure

5.1

(a)

Annual Variation

in

Accidents

district

\section{variation in selected streches 2003-15}

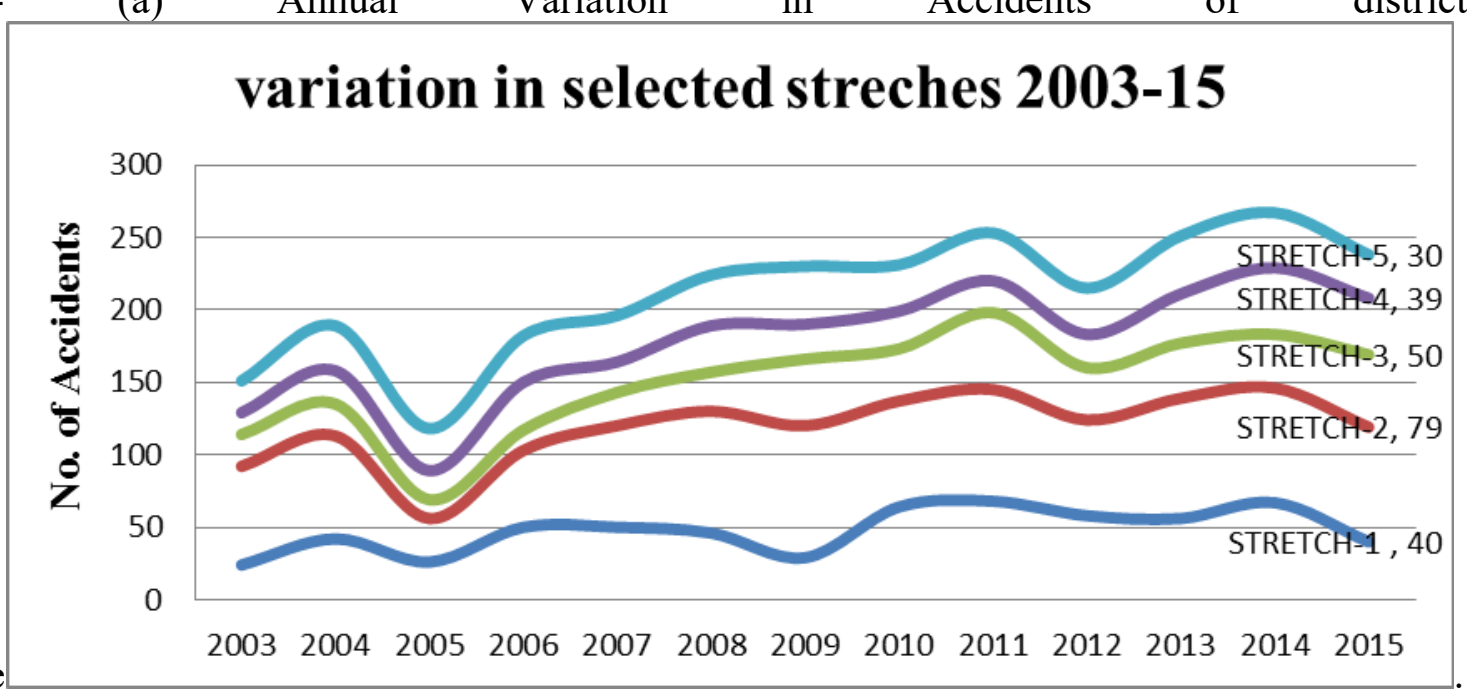

Khargone

Figure 5.1 (b) Annual Variation in Accidents of district of stretches selected for work

\subsection{Annual Variation in Accident Stretch Wise}

(a) Khargone To Kasrawad Road

Figure 5.3 showing variation of accident for stretch -1 i.e. Khargone to Kasrawad road, As the accident are mostly in increasing order, but there are significant drop in accident in year 2005 and 2009 in minor injuries, but as the road facilities increasing average speed increase and all other factor increases the accident. 


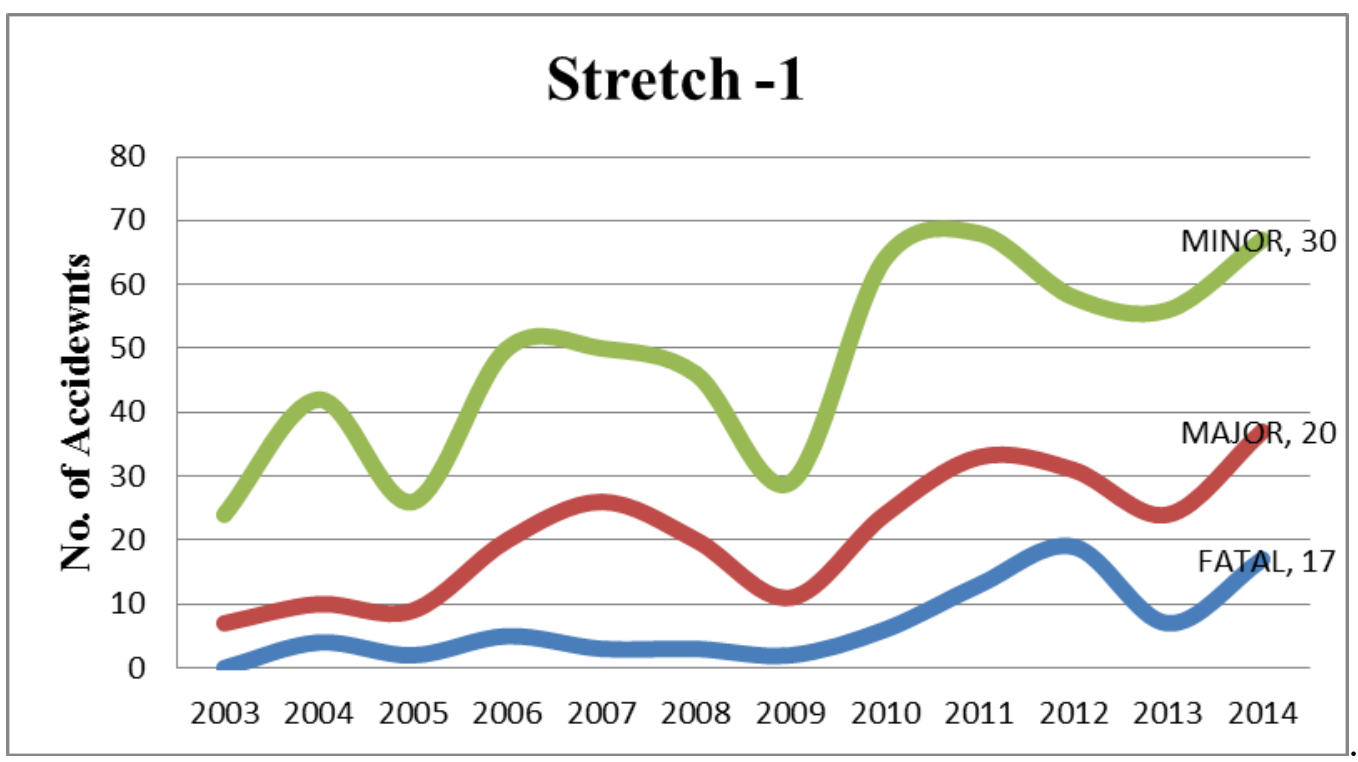

Figure.5.2 -Variation in Accident Stretch -1

\section{(b) Khargone to Sanawad Road}

The Khargone to Sanawad road i.e. stretch -2 is busiest road after proper development of road network and traffic volume is also high, road development started in early of 2005 and Figure 5.4 shows that only year in which accident is minimum and other successive year having increasing order, during 2011-12 rate of minor, major and fatal cases are drop down significantly.

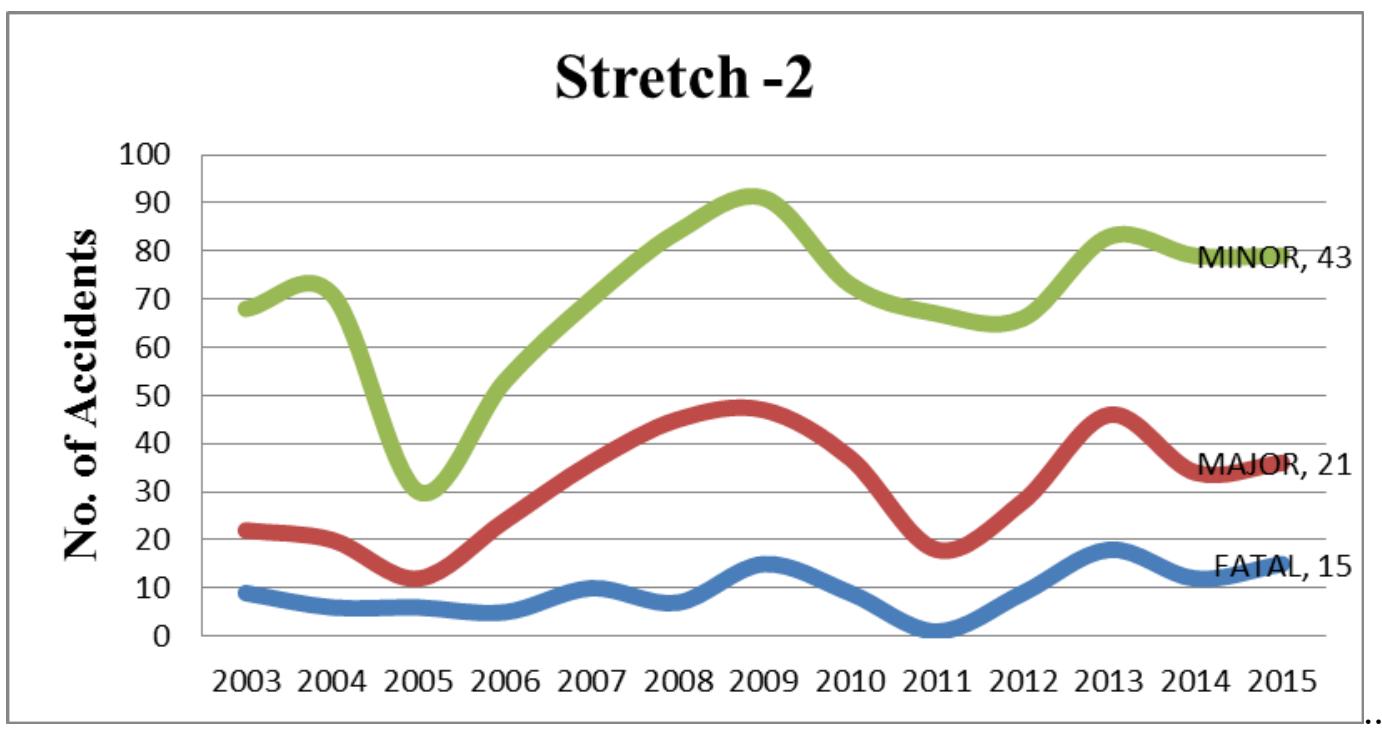

Figure 5.3 -Variation in Accident Stretch -2

(c) Khargone to Julwaniya Road

Figure 5.5 shows the variation in accident year by year from 2003 to 2015. This stretch carry mainly heavy trucks loading vehicle because its connected to the national highway at Julwaniya, the variations shows only drop in accident in year 2010 only, otherwise accident are growing up in minor, major and fatal cases. 


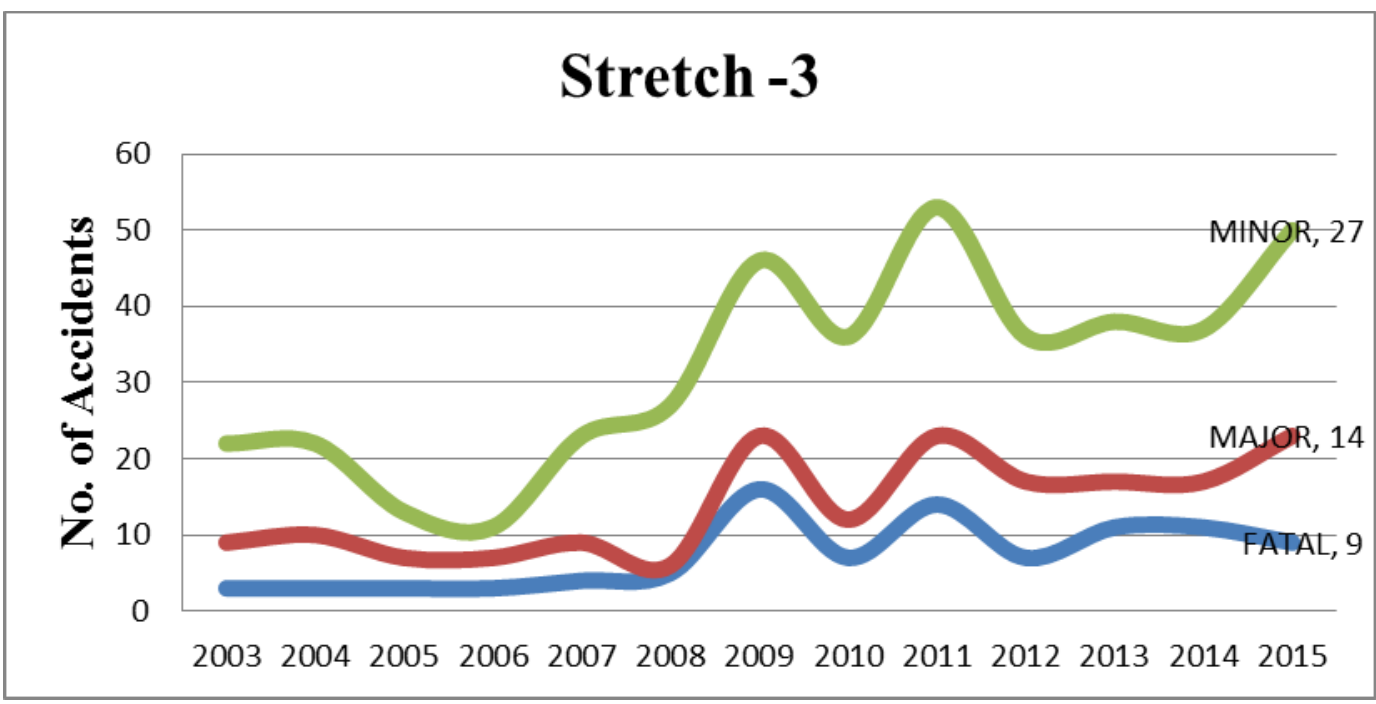

Figure 5.5 -Variation in Accident Stretch -3

(d) Khargone To Bhagwanpura Road :-

Figure 5.6 shows the accident variation of Stretch -4 i.e. Khargone to Bhagwanpura road and stretch carries heavy traffic because road connect two state through Bistan at Mahapal border. This is state highway SH-1, there are accident increasing since 2009 year and major injuries are of increasing order.

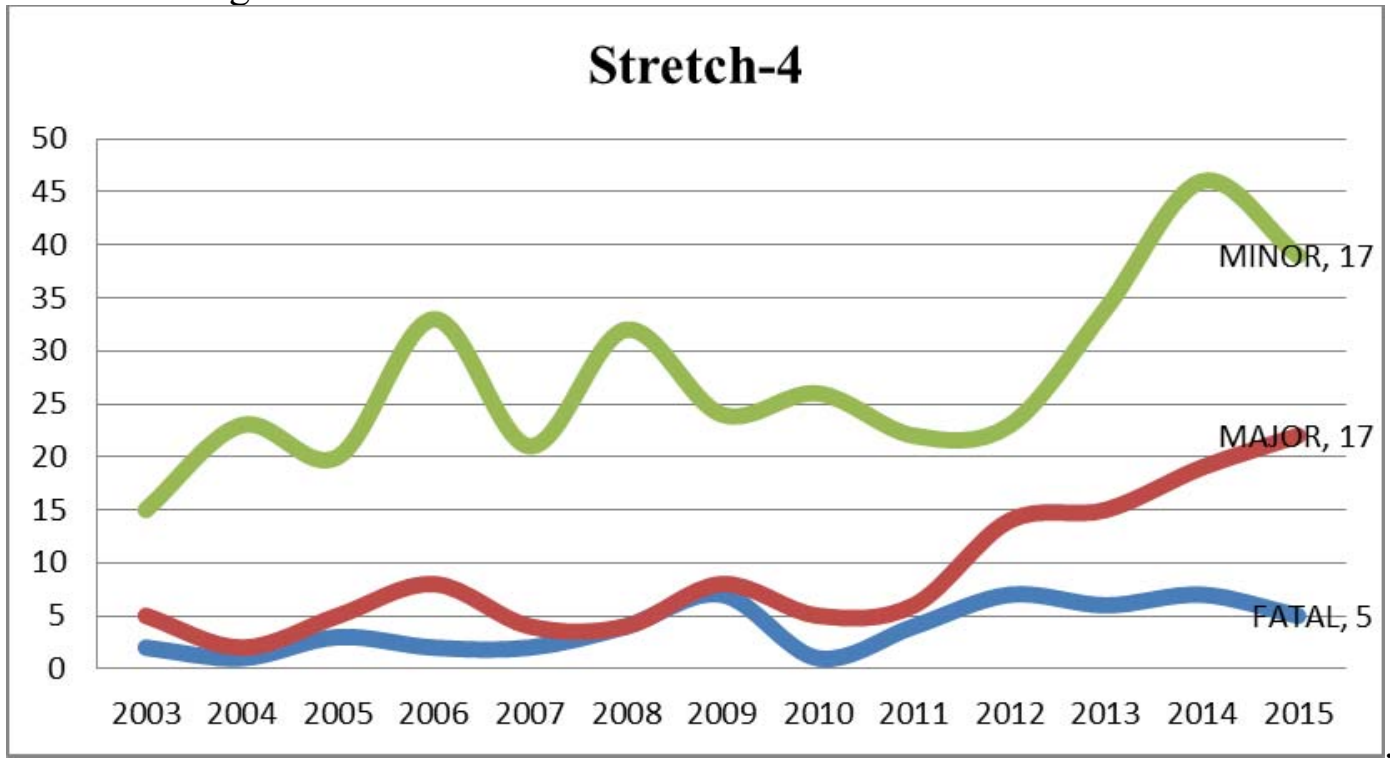

Figure 5.6 -Variation in Accident Stretch -4

\section{(e) Khargone to Bhikangaon Road:}

Figure 5.7 showing the stretch -5 i.e. Khargone to Bhikangaon state highway SH-26. The road connect numbers of block of Khargone, there are mixed Traffic, The accidents increased year by year but there is significant fall in accidents from year 2013 to onward there are barricading by Police provided at Accident Porn Zone so the accident were controlled. 


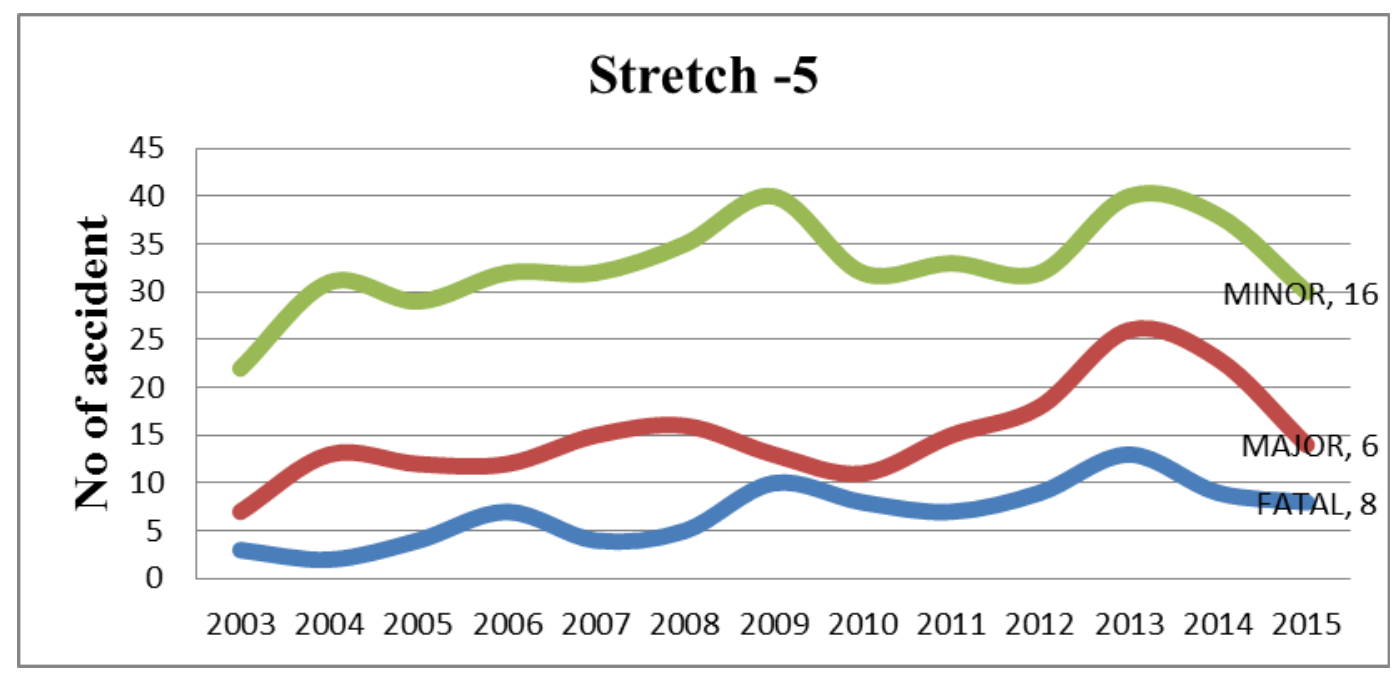

Figure 5.7 -Variation in Accident Stretch -5

\section{(f) Monthly Variation in Accident (2014-2015)}

Figure 5.8 showing the monthly variation of accident of two year i.e. from January 2014 to December 2015, both years most cases of accident reported during month of April. To July, in this summer duration lots of festival and marriage season on pick so that the traffic volume is high.

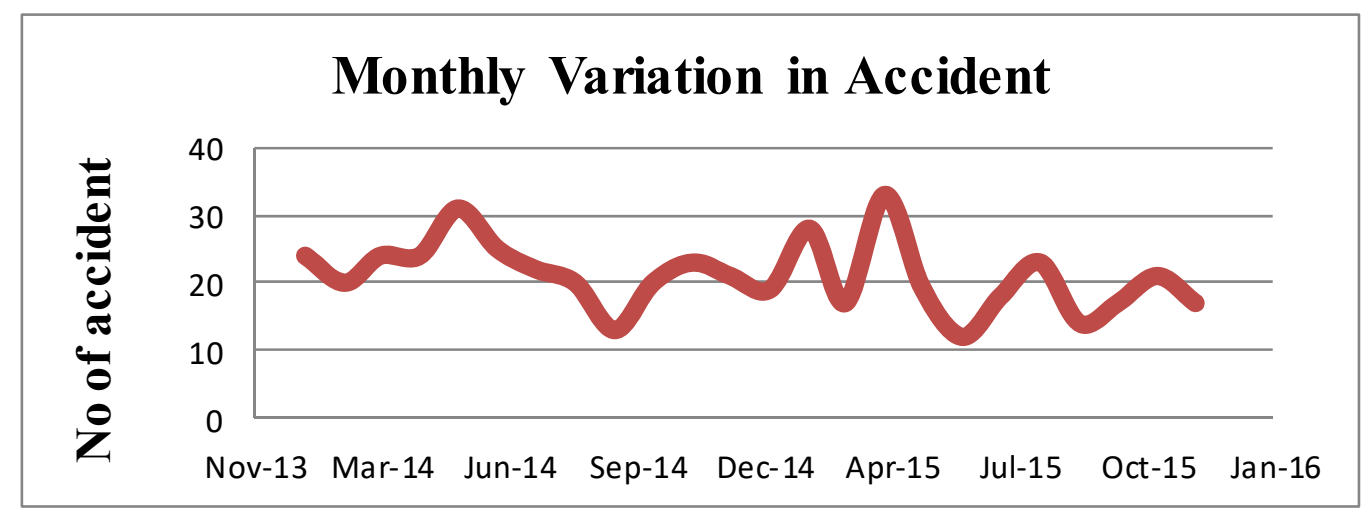

Figure 5.8 Monthly Variation in Accident

(g) Vehicle Involve in Accident during 2006-2015

Figure 5.9 showing result of type of vehicle involved in total accident of all five selected, the pi graph showing that the $40 \%$ of two wheeler are involved in total accident and $47 \%$ vehicles are four wheelers, In the Four Wheelers there are $12 \%$ trucks involve , $14 \%$ of Buses and $21 \%$ four wheelers are car and jeep

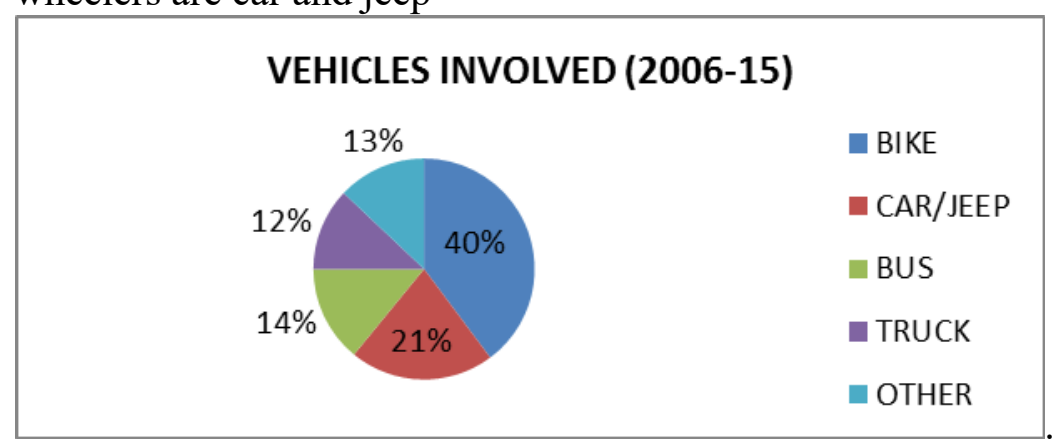


Figure 5.9:- Vehicle Involved in Accidents (2006-15)

\section{VI.ACCIDENT SPOT AND THEIR SAFTEY REQUIRMENT}

\subsection{KHARGONE LOCAL}

Table 6.1:- Khargone local

\begin{tabular}{|l|l|l|l|}
\hline S.NO. & SPOT/STRETCH & PROBLEM & $\begin{array}{l}\text { SAFETY } \\
\text { REQUIREMENTS }\end{array}$ \\
\hline 1 & $\begin{array}{l}\text { Gayatri Mandir } \\
\text { Square }\end{array}$ & $\begin{array}{l}\text { T-Intersection, Narrow Road Margin, } \\
\text { On Road Parking, Heavy Traffic, No } \\
\text { Footpath, Closed Signal System }\end{array}$ & $\begin{array}{l}\text { Junction Development, } \\
\text { Road } \\
\text { Development, Footpath } \\
\text { Required. }\end{array}$ \\
\hline 2 & Diversion Road & $\begin{array}{l}\text { Narrow Road Margin, No Sign For } \\
\text { Median Crossing Unauthorized } \\
\text { Acquisitions Of Shoulder, Heavy } \\
\text { Traffic }\end{array}$ & $\begin{array}{l}\text { Sign System, Shoulder } \\
\text { Clearance, Speed } \\
\text { Barkers At }\end{array}$ \\
\hline 4 & $\begin{array}{l}\text { Navgrah Mandir } \\
\text { Square }\end{array}$ & $\begin{array}{l}\text { Intersection ,Steep Gradient At } \\
\text { Junction,Narrow Road Margin } \\
\text { Undulation On Road, Patches }\end{array}$ & $\begin{array}{l}\text { Junction Requirement, } \\
\text { Overlay Construction } \\
\text { Required. }\end{array}$ \\
\hline 5 & Aurangpura Square & $\begin{array}{l}\text { Heavy Traffic ,Patches, Cracks, On } \\
\text { Road Parking, }\end{array}$ & $\begin{array}{l}\text { Junction Requirements, } \\
\text { Speed Restriction, } \\
\text { Parking Requirement }\end{array}$ \\
\hline
\end{tabular}

\subsection{KHARGONE TO KASRAWAD ROAD}

Table6.2: - Khargone to Kasrawad Route

\begin{tabular}{|c|c|c|c|}
\hline S.NO. & SPOT/ STRETCH & PROBLEM & SAFETY REQUIRMENTS \\
\hline 1 & Bediya (fata) & $\begin{array}{l}\text { Sharp Horizontal Curve, Edge } \\
\text { Drop , Sign ,Small Road } \\
\text { Margin, Wine Outlet }\end{array}$ & $\begin{array}{l}\text { Sign Should Place. } \\
\text { Extra Widening }\end{array}$ \\
\hline 2 & Mengaon & $\begin{array}{l}\text { Staggered Intersection, On } \\
\text { Road Bus Stop, On Road Daily } \\
\text { Vegetable Market, Sign, } \\
\text { Insufficient Road Margin }\end{array}$ & $\begin{array}{lr}\text { Road } & \text { Junction } \\
\text { Improvement, } & \text { Sight } \\
\text { Distance } & \text { Clearance, } \\
\text { Unauthorised } & \text { Acquisition } \\
\text { Clearance } & \end{array}$ \\
\hline 3 & Selani & $\begin{array}{l}\text { Sharp Horizontal Curve ,Sight } \\
\text { Distance Problem , Pavement } \\
\text { Width, Tree On Shoulder }\end{array}$ & $\begin{array}{l}\text { Realignment Requirement } \\
\text { For Short And Easy } \\
\text { Alignment }\end{array}$ \\
\hline 4 & Selani to Sawada & Horizontal Curve , Sight & Realignment, \\
\hline
\end{tabular}




\begin{tabular}{|l|l|l|l|}
\hline & Curve & $\begin{array}{l}\text { Distance , Sign , Marking, } \\
\text { Super Elevation, Steep } \\
\text { Gradient }\end{array}$ & Restrictions, Barricading. \\
\hline 5 & Chhoti Kasrawad & $\begin{array}{l}\text { Sharpe horizontal Curve, Edge } \\
\text { Drop, Wine Outlet ,Narrow } \\
\text { Road Margin }\end{array}$ & $\begin{array}{l}\text { Curve elimination , } \\
\text { Gradient Reduction, Road } \\
\text { side Clearance }\end{array}$ \\
\hline 6 & Kasrawad & $\begin{array}{l}\text { Intersection, Signal, Sign, Road } \\
\text { Margin, Shoulder On Street } \\
\text { Parking, Garages, Hospital, } \\
\text { Sharp Curve. }\end{array}$ & $\begin{array}{l}\text { Junction improvement, } \\
\text { Shoulder Clearance, } \\
\text { parking facility } \\
\text { development. }\end{array}$ \\
\hline
\end{tabular}

\subsection{KHARGONE TO JULWANIYA}

Table6.3: - Khargone to Julwaniya Road

\begin{tabular}{|c|c|c|c|}
\hline $\begin{array}{l}\text { S.N } \\
0\end{array}$ & SPOT/STRETCH & PROBLEM & $\begin{array}{l}\text { SAFETY } \\
\text { REQUIRMENTS }\end{array}$ \\
\hline 1 & Barud Fata & $\begin{array}{l}\text { T-Intersection, Bus Stop, On Road } \\
\text { Bus Stop, Narrow Road Width At } \\
\text { Junction, Sight Distance }\end{array}$ & $\begin{array}{lr}\text { Separate Space } & \text { For Bus } \\
\text { Stop, } & \text { Margin } \\
\text { Improvement. } & \end{array}$ \\
\hline 2 & Nandgaon & $\begin{array}{l}\text { Trees On Shoulder, On Road Bus } \\
\text { Stop,No Sign For Crossing, Narrow } \\
\text { Bridge , Tress On Shoulder Edge } \\
\text { Drop At Intersection }\end{array}$ & \begin{tabular}{lrr} 
Junction & \multicolumn{2}{r}{ Improvement, } \\
Widening Of & Bridge, \\
Railing On & Bridge \\
\multicolumn{2}{l}{ Shoulder Maintenance }
\end{tabular} \\
\hline 3 & Banihar & $\begin{array}{l}\text { Unnecessary Speed Barkers, Trees } \\
\text { On Shoulder, On Road Bus Stop, } \\
\text { Pole On Shoulder, No Regulatory } \\
\text { And Informatory Sign }\end{array}$ & $\begin{array}{ll}\text { Shoulder } & \text { Maintenance, } \\
\text { Junction } & \text { Improvement, } \\
\text { Sign } & \end{array}$ \\
\hline 4 & Oon & $\begin{array}{l}\text { T-Intersection, ,Temple , Narrow } \\
\text { Culvert Bridge, Road Margin, On } \\
\text { Road Bus Stop, }\end{array}$ & $\begin{array}{l}\text { Bridge Maintenances , } \\
\text { Junction Improvement Sign }\end{array}$ \\
\hline 5 & Jamothi Fata & $\begin{array}{l}\text { Combination Of Horizontal And } \\
\text { Vertical Summit Curve, Sharp Turn, } \\
\text { Steep Gradient, Narrow Culvert }\end{array}$ & $\begin{array}{l}\text { Alignment Improvement } \\
\text {,Sight Distance Clearance } \\
\text {,Sign }\end{array}$ \\
\hline
\end{tabular}




\begin{tabular}{|l|l|l|l|}
\hline & & Bridge,Sight Distance & \\
\hline 6 & Talakpura & $\begin{array}{l}\text { Vertical Summit Curve And T- } \\
\text { Intersection Narrow Road Margin }\end{array}$ & $\begin{array}{l}\text { Alignment Improvement } \\
\text {,Flat Gradient ,Junction } \\
\text { Improvement }\end{array}$ \\
\hline
\end{tabular}

6.4 KHARGONE TO BHAGWANPURA

Table 6.4 Khargone to Bhagwanpura Road

\begin{tabular}{|l|l|l|l|}
\hline S.NO. & SPOT/STRETCH & PROBLEM & SAFETY REQUIRMENT \\
\hline 1 & Tata Showroom & $\begin{array}{l}\text { Narrow Culvert Bridge without } \\
\text { Railing,Cracks on Road ,Sight } \\
\text { Distance, On Street Parking }\end{array}$ & $\begin{array}{l}\text { Reconstruction of bridge } \\
\text { Road Side Clearance , } \\
\text { Sight Distance Clearance }\end{array}$ \\
\hline 2 & $\begin{array}{l}\text { Stretch 4.5 Km To } \\
5 \mathrm{Km}\end{array}$ & $\begin{array}{l}\text { Sharp Horizontal Curve , Sight } \\
\text { Distance Problem }\end{array}$ & $\begin{array}{l}\text { Inner Side Clearance } \\
\text {,Road width } \\
\text { Improvements, Sign }\end{array}$ \\
\hline 3 & Jamli & $\begin{array}{l}\text { T-intersection at both side of } \\
\text { village, Road Margin }\end{array}$ & $\begin{array}{l}\text { Junction Improvement } \\
\text {,Shoulder Clearance, Edge } \\
\text { Drop }\end{array}$ \\
\hline 5 & $\begin{array}{l}\text { Bijasani } \\
\text { Restaurant curve }\end{array}$ & $\begin{array}{l}\text { Sharp horizontal curve , poor } \\
\text { condition of bridge , narrow road at } \\
\text { curve }\end{array}$ & $\begin{array}{l}\text { Bridge Improvement } \\
\text {,Overlay construction } \\
\text {,Width improvement, Flat } \\
\text { gradient towards Bistan }\end{array}$ \\
\hline 6 & Ghatti Village & $\begin{array}{l}\text { Curved road , Narrow Pipe culvert } \\
\text { bridge }\end{array}$ & $\begin{array}{l}\text { Bridge Improvement, Inner } \\
\text { Clearance }\end{array}$ \\
\hline
\end{tabular}

\subsection{KHARGONE TO BHIKANGAON}

Table6.5 Khargone to Bhikangaon Road

\begin{tabular}{|c|c|c|c|}
\hline S.NO & SPOT /STRETCH & PROBLEM & SAFETY REQUIRMENT \\
\hline 1 & $\begin{array}{l}\text { Gopalpura } \\
\text { Magriya }\end{array}$ & $\begin{array}{l}\text { T-Intersection, Sign ,Steep } \\
\text { Gradient From Village Road, } \\
\text { Sight Distance Problem, On } \\
\text { Road Bus Stop, Tress On }\end{array}$ & $\begin{array}{l}\text { Junction Improvement, } \\
\text { Shoulder Clearance, Speed } \\
\text { Restriction. }\end{array}$ \\
\hline
\end{tabular}




\begin{tabular}{|c|c|c|c|}
\hline & & Shoulder & \\
\hline 2 & Ghughriyakhedi & $\begin{array}{l}\text { On Road Bus Stop, On Road } \\
\text { Weekly Market, Staggered } \\
\text { Intersection , No Informatory } \\
\text { And Regulatory Sign }\end{array}$ & $\begin{array}{l}\text { Shoulder Clearance, } \\
\text { Junction Improvements, } \\
\text { Road Margin, Speed } \\
\text { Restriction. Width At } \\
\text { Junction Point }\end{array}$ \\
\hline 3 & Bilali & $\begin{array}{l}\text { Staggered Intersection } \quad \text {,Edge } \\
\text { Drop, Signs }\end{array}$ & $\begin{array}{l}\text { Junction Improvement, } \\
\text { Tress Clearance Edge Drop, } \\
\text { Speed Restriction. }\end{array}$ \\
\hline 4 & Behrampur Tema & $\begin{array}{l}\text { T-intersection, on Road Bus } \\
\text { stop, }\end{array}$ & $\begin{array}{l}\text { Junction Improvement, } \\
\text { Tree Clearance ,Edge Drop } \\
\text {,Speed Restriction }\end{array}$ \\
\hline 5 & Lalani Timarni & $\begin{array}{lll}\text { On Road Bus Stop , } & \text { Steep } \\
\text { Gradient , Crossing , } & \text { Road } \\
\text { Patches } & & \end{array}$ & $\begin{array}{l}\text { Bus Stop, Side Clearance, } \\
\text { Overlay Requirement }\end{array}$ \\
\hline 6 & Surva & $\begin{array}{l}\text { On Road Bus Stop, Crossing, } \\
\text { Sight Distance ,Speed Sign }\end{array}$ & $\begin{array}{l}\text { Inner Side Clearance, } \\
\text { Junction Improvement. }\end{array}$ \\
\hline 7 & Bhikangaon & $\begin{array}{l}\text { T And Staggered Junction, No } \\
\text { Shoulder At Junction, No Road } \\
\text { Margin, On Shoulder Market }\end{array}$ & $\begin{array}{l}\text { Junction Improvement, } \\
\text { Median Requirement, Speed } \\
\text { Limit, Shoulder Clearance }\end{array}$ \\
\hline 8 & $\begin{array}{l}\text { Temla To } \\
\text { Bhikangaon Stretch }\end{array}$ & $\begin{array}{l}\text { Sharp Horizontal Curve, Steep } \\
\text { Gradient , Bridge , Staggered } \\
\text { Junction, Temple On Shoulder, } \\
\text { Trees On Shoulder }\end{array}$ & $\begin{array}{l}\text { Gradient elimination, Inner } \\
\text { side Clearance, Junction } \\
\text { improvement }\end{array}$ \\
\hline
\end{tabular}

\section{CONCLUSION AND RECOMMONDATIONS}

\subsection{CONCLUSION}

1. The available literature on accident Analysis shows $77.44 \%$ of accident due to drivers' faults, $4.266 \%$ due to driver of other vehicle, and $1.71 \%$ due to Road side features.

2. CAGR report shows $53.70 \%$ on rural road and $46.30 \%$ on Urban Road in year 2014 .

3. All of stretches mainly having problem of accident at junction, road side feature, Narrow bridges, sharp curves, On road Bus stop.

4. Khargone to Kasrawad Stretch having largest number of accident rate while the Julwaniya route has minimum. 
5. Khargone town its self having 5 major accident spots, there is heavy mixed trac, 4 spots out of 5 are junction point ,mainly there are narrow road margin, on street parking, no signal system and diversion road having problem of median crossing.

6. Khargone to Kasrawad route having problem related to sharp curves, inner side clearance requirement at curves, on road bus stops, shoulder clearance and junction improvement. There are mengaon and kasrawad having problems related to manmade features like un-wanted speed barkers, un authorised space occupy by temporary on road daily market

7. Khargone to Julwaniya route having problem of on road bus stoppage, staggered no sign intersections, narrow bridge sharp horizontal curve and steep gradient, the crakes and patches are not on every accident spot/stretch, few spots having trees on shoulders and electric pole. Jamothi fata and Talakpura stretch has large length of curves.

8. Khargone to Bhagwanpura state highway is suffering from bad bridge condition, narrow and railing less bridge also includes intersections, poor surface-condition, sight distance and edge drops and trees on shoulder.

9. Khargone to bhikangaon route 7 spots out of 8 spots having problem no sign junction or intersections, 3 spots having problem of sight distance restriction and edge drop too.

10. Khargone to Sanawad road has been started for the widening of road.

\subsection{RECOMMONDATIONS}

\subsubsection{KHARGONE TOWN}

(a) Gaytri mandir Triangle: The spot carries two state highway and other district road traffic to main town Khargone, this is only spot which required a proper signal system, there already signal system installed but not in working condition, road margin are need to be improve and there should be footpath for the passengers, because bus stand just at 200 meters away.

(b) Diversion Road : There are no proper sign system is available for the road user to guide them properly while negotiating the median, shoulder clearance required because there is main market i.e. radhawallabh which carries large amount of traffic and speed barkers should be placed before median gap.

(c) Navgrah Mandir square: There are large undulations at intersection point and road surface is very poor lots of patches and crakes are there road from main town meet at positive gradient. There should be laying of overlay and junction width improvement.

(d) Aurangpura Square : The pavement surface is poor, lots of crakes on and patches on road surface, location has small on road market, lots of space is occupied by on street parking and road connected Julwaniya state highway heavy as well as light vehicle traffic is high. Overlay construction and roadside clearance is required for sufficient road margin, speed restriction by speed breakers can be achieved.

(e) Bistan Naka: This spot having T- intersection, due to on street parking of tempo and auto effective width of road is reduced the spot on Bistan road highway also main playground, polytechnic college and P.G. college are nearby situated. There should be improvement of parking facility, increased road margin and shoulder clearance is required.

\subsubsection{KHARGONE TO KASRAWAD ROAD}

(a) Bediya (fata): at this spot of kasrawad highway sharp horizontal curve with steep gradient is provided, road required extra widening and also there shoulder be speed limit restriction sign and speed breaker should be installed to minimize accidents.

(b) Mengaon : There are staggered intersection one of them having no minimum sight distance at intersection, and there are steep gradient at intersection point, right now to minimize the speed 
there are barricades are placed but there should be informatory sign provide and speed breaker should be place at both side of intersection point.

(c) Selani : There is sharp horizontal curve situated having no inner clearance and no proper sight distance, there are tree at out edge of road, in order to minimize accident, the road should be realigned, so sort , easy and economical alignment can be stabilize.

(d) Selani to Sawda Curve: There is large horizontal and vertical combination is placed. There are extra widening requirement, the super elevation should be redesign, one for all problem the alignment should be redesigned to minimize accident rate.

(e) Chhoti Kasrawad: There are large horizontal curve is situated, the curve should be eliminate to provide proper and sufficient sight distance at design speed.

(f) Kasrawad : there are junction improvement is required, there should be increase in road width as well as road margin, there are on street parking which reduces the effective width so there shoulder clearance is required to provide proper road width, another problem is sharp, narrow horizontal curve, there also un authorised acquisition by temporary market, that market should be replace.

\subsubsection{KHARGONE OT JULWANIYA}

(a) Barud fata: There is $\mathrm{T}$ junction, the spot having on road bus stop with small road margin. There should be separate space for bus stop so that the sight distance can't obstruct and width of road at $\mathrm{T}$ junction should be improved to eliminate edge drop.

(b) Nandgaon bagud : The spot is junction point of village road and state highway, there should be place informatory sign and speed breaker, another problem is narrow bridge, which required to be reconstruct as per state highway standards

(c) Banihar: There are on road bus stop with speed breakers but no sign for speed breakers, sign should be provide and breaker should be marked again. Junction point required sufficient width of road pavement to eliminated edge drop.

(d) Oon: There are staggered intersection at end of valley curve, the bridge should be reconstruct as well as required re-alignment to provide proper sight distance, width of road pavement at junction point should be increase to eliminate edge drop.

(e) Jamothi fata: The combination of sharp horizontal and steep vertical curve requires realignment, There are speed limit restrictions provide by speed breakers or by barricading to minimize speed of traffic.

(f) Talakpura: there are junction is situated at peak of summit curve so the spot does not offer any sight distance at vertical curve. The apex of curve will be reduced by realignment.

\subsubsection{KHARGONE TO BHAGWANPURA}

(a) Tata showroom: The spot having narrow culvert bridge in damage condition and road surface also poor, bridge should be reconstruct with new alignments of road and bridge to improve sight distance and carry traffic and design speed.

(b) Stretch $4.5 \mathrm{~km}$ to $5 \mathrm{Km}$ : There is sharp horizontal curve which does not offered minimum sight distance at design speed also there is requirement of extra widening at curve, there are large edge drop on outer edge at curve.

(c) Jamli: Staggered intersection having insufficient required at curve so there proper informatory sign and speed breakers should be provided to reduce the rate of accidents.

(d) Stretch $7 \mathrm{~km}$ to $8 \mathrm{~km}$ :- There is sharp horizontal curve is placed with narrow bridge, the width of bridge should be increase as per Standard of State Highway and inner side clearance required for sight Distance also widening at curve is required.

(e) Bijasani Restaurant Curve: - A sharp horizontal curve with damaged narrow bridge is situated. The Road pavement condition is poor, consisting of large cracks and patches. There also a problem the 
road from Bistan side coming with steep gradient on narrow bridge, so flat gradient required to reduce risk of accident, overlay construction required and reconstruction of bridge with proper desired width. If flat gradient is not provided then speed restricting sign with suitable speed breakers should be place.

(f) Ghatti village: - Spot having damaged narrow bridge without railing, and sharp curve, in order to reduce the accident the spot requires inner side clearance for sight distance and repairing damaged bridge more prefer re-construction of bridge because existing bridge is narrow.

\subsubsection{KHARGONE TO BHIKANGAON}

(a) Gopalpura to Magriya : stretch between Gopalpura to Magriya having T intersection , on road bus stop and tress on shoulder, the stretch Required junction improvement in the form of proper width at intersection, shoulder clearance, speed restriction through speed breakers in order to provide sufficient sight distance at intersection.

(b) Ghughriyakhedi: Spot having problems due to Intersection, on road Bus stop and no informatory signs, the reduction is cases of accident can be achieved by proper sign placement, width of road at intersection and speed restriction by speed breakers before intersection.

(c) Bilali: There are no informatory and regulatory sign at staggered intersection as well as problem of edge drop at intersection, there should be proper sign and road width improvement at intersection requires reducing accident.

(d) Behrampur Tema: there is on Road bus stop and T-intersection, on road bus stop obstruct the sight distance ahead and insufficient width of intersection increases edge drop.

(e) Lalani Timarni : The stop having on road bus stop, and intersection on with main State Highway comes with steep gradient and curved road, road surface is poor, the spot should be reconstruct and flat gradient can be achieved by realignment of road.

(f) Surva: - Location has crossing point and On Road bus stop, so there is problem of sight distance restriction. In order to provide proper Sight Distance inner Side clearance and junction width enhancement required.

(g) Bhikangaon: Bhikangaon village having $\mathrm{T}$ and Staggered junction, main market has maximum space occupy by on road temporary shop, Road margin are not sufficient and shoulder are not clear. Intersections having not sufficient space at junction, junctions should be provided with island and road margins should be increase by shoulder clearance.

(h) Temla to Bhikangaon: The stretch having Sharp curve , Steep Gradient ,Staggered and tress on shoulder. There is problem of Sight Distance eliminated by inner clearance by extra widening and trees clearance.

\section{REFERENCES}

[1] Global Road Safety Partnership Annual Report (2011) . http://www.grsproadsafety.org/sites/grsp.drupalgardens.com/files.

[2] Accidental deaths in ndia (2014), National Crime Records Bureau, Ministry of Home Affairs, Government of India, New Delhi.

[3] New geometric design consistency model based on operating speed Profiles for road safety evaluation, Accident Analysis and Prevention Article in press AAP-2915 pp.1-10

[4] Sivakumar, .Krishnaraj(2012),Road Traffic Accidents (RTAs) Due To Drunken Driving In India, Challenges In Prevention international journal of research in management and Technology,ISSN:2249 9563 VOL. 2, pp.401-406

[5] Hassan and Aty(2012) Exploring the safety implications of young drivers attitudes and perceptions, Accident Analysis and Prevention Vol.43 pp.45-65. 
[6] The Effect of Pavement Condition on Traffic Safety: A Case Study of Some Federal Roads in Bauchi State A. Mohammed1, S. Y. Umar,D. Samson and T. Y. Ahmad. Department of Civil Engineering, Abubakar Tafawa Balewa University, Bauchi,P. M. B 0248 Bauchi, Bauchi State Nigeria.

[7] Jinsun and Doohee(2003)Run-off-Roadway Accident Frequency Models By Type of Accident Severity Journal of the Eastern Asia Society for Transportation Studies, Vol.5, October, 2003.

[8] Longo et al(1999)The prevalence of alcohol, cannabinoids, benzodiazepines and stimulants amongst injured drivers and their role in driver culpability, Accident Analysis and Prevention Vol.32 pp.623 - 632

[9] Campus et al.,(2011) The effect of the new traffic law on drinking and driving in Sao Paulo, Brazil Accident Analysis and Prevention Vol.50 pp.622-627.

[10] Labat et al.,(2003) Prevalence of psychoactive substances in truck drivers in the Nord-Pas-deCalais region (France), Forensic Science International Vol.174 pp.90-94

[11] Holmgren et al.,(2003) Alcohol and drugs in drivers fatally injured in traffic accidents in Sweden during the years 2000-2002 Forensic Science International Vol.151 pp.11-17.

[12] Labat et al.,(2003) Prevalence of psychoactive substances in truck drivers in the Nord-Pas-deCalais region (France), Forensic Science International Vol.174 pp.90-94.

[13] Rio et al (2001) Alcohol, illicit drugs and medicinal drugs in fatally injured drivers in Spain between 1991 and 2000Forensic Science International vol.127 pp.63-70.

[14] Keall et al(2002)The influence of alcohol, age and number of passengers on the night-time risk of driver fatal injury in New Zealand Accident Analysis and Prevention vol. 36 pp.49-61.

[15] Prabhakhara $\mathrm{n}$ et al.,(2012 )Impairment of a speed management strategy inyoung drivers under high cognitive work load Accident Analysis and Prevention Vol.47 pp.24-29

[16] Aarts and Schagen(2006) Driving speed and the risk of road crashes: A review, Accident analysis is and Prevention Vol.38 pp.215-224.

[17] Golob andRecker(2003) Journal of Transport Engineering Vol.4 pp-129.

[18] Houquani et al.,(2012) Sleep related collisons in United Arab Emirates, Accident Analysis and Prevention Vol.50 pp.1052-1055.

[19] Blower et al.,(1998) Fatalities and Injuries in Truck crashes by Time of day, University of Michigan Transport Research Institute 1998.

[20] Tornros and Boiling(2005)Mobile phone use-Effects of handheld and hands free phones ondriving performance Accident Analysis and Prevention Vol.37 pp.902-909.

[21] Bendak(2005) Seat belt utilization in Saudi Arabia and its impact on road accident injuries. Accident analysis and prevention Vol.2 pp.367-71.

[22] Jamalludin(2012). The Effective Way to Create Awareness among Express Bus Passenger in Using Seatbelt within West Coast Malaysia American ,International Journal of Contemporary Research Vol. 2 No. 9.

[23] Asa et al(1999)The prevalence of non-standard helmet use and head injuries among motorcycle riders Accident Analysis and Prevention vol.31 pp. 229-233

[24] Gabel (1999) Use of helmet and injury prevention Accident Analysis and Prevention Vol.21 pp. 245-246.

[25] Gabel(1999) Use of helmet and injury prevention Accident Analysis and Prevention Vol.21 pp. 245-246

[26] Sreedharan(201)0 Determinants of safety helmet use among motorcyclists in Kerala, India Journal of Injury and Violence Research, Vol. 2, No 1 (2010).

[27] Forest et al(2009) Influence of Roadway Surface Discontinuities' on Road Safety TRB ISSN: 0097- 8515

[28] Jinsun and Doohee(2003)Run-off-Roadway Accident Frequency Models By Type of Accident Severity Journal of the Eastern Asia Society for Transportation Studies, Vol.5, October, 2003. 